\title{
Very late relapse of chronic myelogenous leukemia following stem cell transplant
}

\begin{abstract}
Chronic myelogenous leukemia (CML) is a myeloproliferative neoplasm of the bone marrow that is associated with the Philadelphia chromosome, which creates the fusion BCR-ABL tyrosine kinase. Prior to development of tyrosine kinase inhibitors such as imatinib, the standard of care for treatment in certain clinical situations was hematopoietic stem cell transplant. We present a case of a 61 year-old male with a history of CML who received a stem cell transplant 20years prior. He now had developed an increased white blood cell count and was found to have recurrence of CML in his bone marrow and via peripheral blood analysis for BCR-ABL. Through further evaluation it was found that the recurrence was genetically the same as his original malignancy and not a de novo evolution of his sibling donor graft cells. He was trialed on several tyrosine kinase inhibitors following his diagnosis but had complications with one and progression on another. He was eventually given a donor leukocyte infusion to combat his disease which improved his status and was without complication. This case demonstrates a rare but known entity of very later recurrence of CML following stem cell transplant.
\end{abstract}

Volume I Issue I - 2015

\author{
Matthew Richards, James Gajewski
}

Oregon Health \& Science University, USA

Correspondence: Matthew Richards, Oregon Health \&

Science University, USA, Email richamat@ohsu.edu

Received: January 06, 2015 | Published: April 07, 2015
Abbreviations: CML, chronic myelogenous leukemia; TKI, tyrosine kinase inhibitor; DLI, donor leukocyte infusions

\section{Introduction}

Chronic myelogenous leukemia (CML) is a myeloproliferative neoplasm that originates in a pluripotent bone marrow stem cell and is associated with the product of the Philadelphia chromosome, the BCR-ABL1 fusion gene. ${ }^{1}$ This gene product creates a constitutively active tyrosine kinase resulting in non-cytokine dependent cell growth. The disease can be categorized as in one of three phases: chronic phase, accelerated phase, or blast phase. The natural history of the disease is progression from the chronic phase to blast crisis in three to five years. ${ }^{2}$ Until the development of novel therapies for the disease, the standard of care for treatment of CML was hematopoietic stem cell transplant in the early chronic phase. Initially, attempts were made with autologous transplants utilizing cryopreserved marrow from the patient; however, this resulted in rapid establishment of chronic phase soon thereafter. ${ }^{3}$ This was utilized as a stopgap in blast crisis to reestablish chronic phase. The first allogeneic transplants for CML occurred in Seattle utilizing identical twins bone marrow in four patients with sustained hematologic remission at 31 months. ${ }^{4}$ This led to a rise in allogeneic transplants mostly utilizing matched sibling donors which ultimately became the standard therapy for eligible patients. At the turn of the century, development of the oral tyrosine kinase inhibitor (TKI), imatinib, created a new era in therapy for CML as a excellent option for patients in chronic phase. ${ }^{5}$ With that development, as well as the newer generation oral TKI drugs currently available, hematopoietic stem cell transplantation fell out of favor as the safety profile for these new medications greatly outweighed the risk of transplant.

The outcomes for stem cell transplant in patients with CML have improved over time with survival rates increasing over time. However, there has not been much progress for survival in patients who were transplanted later in their disease. Additionally, the type of preparative regimen and timing of transplant in the course of the disease has an effect on overall survival favoring early course and reduced intensity regimen. Predictors for relapse throughout the follow up of patients include reduced intensity preparative regimen, unrelated donor, and older age. ${ }^{6}$ Prior to development of oral TKI therapy, donor leukocyte infusions (DLI) were the therapy of choice in relapsed CML posttransplant. This has largely been replaced by oral therapy in the postTKI era though there are situations in which DLI can be utilized.

\section{Case presentation}

The patient is a 61year old male with a history of, hypertension, coronary artery disease, and chronic myelogenous leukemia that underwent matched sibling allogeneic bone marrow transplant at age 41 for early chronic phase disease, the standard of care at that time. His pre-transplant treatment course had only included hydroxyurea. He underwent a preparative regimen of total body irradiation with a dose of 1175 rads followed by two doses of cyclophosphamide $60 \mathrm{mg} /$ $\mathrm{kg}$ with a course of cyclosporine and short course of methotrexate for immunosuppression and GVHD prophylaxis. He represented with a referral due to an elevated white blood cell count to $22 \times 10^{5}$ cells/ $\mathrm{uL}$. A bone marrow biopsy demonstrated a chronic myeloproliferative neoplasm with a hypercellular marrow at $80 \%$ and few blasts (1\%) and a peripheral myeloid predominance with a shift toward immaturity.

Further genetic testing confirmed that this was a recurrence of the patient's original chronic myelogenous leukemia and not a de novo disease from his donor cells. An immunologic workup as well indicated that his lymphocytes were of donor origin and his myeloid cells were of recipient origin. He was started on nilotinib at the time of diagnosis and had appropriate biochemical response within 6months. He continued on therapy with continued morphologic and biochemical response. However out of concern for accerlerated atherosclerosis due to interval coronary artery stent placement and discovery of carotid and peripheral vascular atherosclerosis, nilotinib was discontinued 2 years after initiation. He was started on low dose bosuntinib but this was quickly discontinued due to gastrointestinal toxicity complicated by dehydration and kidney injury that persisted but did not require 
dialysis. Due to lowered baseline renal function, it was decided to treat the patient with donor lymphocyte infusions for management of his recurrence. 3 years after recurrence the patient received $1.13 \times 10^{7}$ $\mathrm{CD} 3+$ cells $/ \mathrm{kg}$ without complication.

\section{Discussion}

Very late recurrence of chronic myelogenous leukemia is a rare entity with very little clinical data. Good data is available to suggest mortality rates compared to the general population in patients 2,5 , and 10years. ${ }^{7}$ However, what little data that is available regarding outcomes after a decade shows that mortality rates return to that of the general population after 14years post-transplant. ${ }^{8}$ Here we present a rare case of very late relapse twenty years after transplant, the second longest period of relapse reported in the literature. Given the rarity of these very later recurrences, it brings up the intriguing premise of what mechanisms are at play that allows a leukemic cell to result in relapse decades later. The rarity of this condition limits the amount of data and cases available for evaluation and study. Several theories are possible including a prolonged state of dormancy in the leukemia stem cell or immune surveillance which is able to resist significant proliferation. The premise of quiescent cells relates to leukemic stem cells which reside in the bone marrow metabolically inactive resistant to radiation and chemotherapy. ${ }^{9}$ These factors result in a state of prolonged disease clearing until an undetermined event results in rise in the disease. As these events are poorly understood, the ability to intervene is limited.

Complete cure of hematologic malignancies and variety of solid tumors has proven to be an elusive entity. Due to the ability of cancer and leukemia stem cells to elude immune or chemotherapy clearance, the risk of recurrence is always present. This risk is even present many years after remission. Until control of these quiescent progenitors can be obtained, complete control of disease will not be achieved. In the future, it will take targeted therapy to remove these cells and eliminate the possibility of regeneration of diseased cells.

\section{Acknowledgements}

None.

\section{Conflict of interest}

The author declares no conflict of interest.

\section{References}

1. Vardiman JW. Chronic myelogenous leukemia, BCR-ABL1+. Am J ClinPathol. 2009;132(2):250-260.

2. Sawyers CL. Chronic myeloid leukemia. $N$ Engl $J$ Med. 1999;340(17):1330-1340.

3. Pavlu J, Szydlo RM, Goldman JM, et al. Three decades of transplantation for chronic myeloid leukemia: what have we learned? Blood. 2011;117(3):755-763.

4. Fefer A, Cheever MA, Thomas ED, et al. Disappearance of Ph1positive cells in four patients with chronic granulocytic leukemia after chemotherapy, irradiation and marrow transplantation from an identical twin. N Engl J Med. 1979;300(7):333-337.

5. Druker BJ, Talpaz M, Resta DJ, et al. Efficacy and safety of a specific inhibitor of the BCR-ABL tyrosine kinase in chronic myeloid leukemia. N Engl J Med. 2001;344(14):1031-1037.

6. Gratwohl A, Brand R, Apperley J, et al. Allogeneic hematopoietic stem cell transplantation for chronic myeloid leukemia in Europe 2006: transplant activity, long-term data and current results. An analysis by the chronic leukemia working. Haematologica. 2006;91(4):513-521.

7. Party of the european group for blood and marrow transplantation (EBMT). Haematologica. 91(4):513-521.

8. Sekhri A, Liu D, Rasul M, et al. Very late relapse of chronic myelogenous leukemia after allogeneic bone marrow transplantation. Leuk Res. 2009;33(9):1291-1293

9. Goldman JM, Majhail NS, Klein JP, et al. Relapse and late mortality in 5-year survivors of myeloablative allogeneic hematopoietic cell transplantation for chronic myeloid leukemia in first chronic phase. $J$ ClinOncol. 2010;28(11):1888-1895.

10. Saito Y, Kitamura H, Hijikata A, et al. Identification of therapeutic targets for quiescent, chemotherapy-resistant human leukemia stem cells. Sci Trans Med. 2010;2(17). 